\title{
Comparação entre o polarímetro de varredura a laser, a tomografia de coerência óptica 1 e o Stratus-OCT na detecção da perda axonal da atrofia em banda do nervo óptico
}

\author{
Comparison of scanning laser polarimetry, optical coherence tomography 1 \\ and Stratus optical coherence tomography for the detection of axonal loss \\ in band atrophy of the optic nerve
}

Bruno Campelo Leal ${ }^{1}$

Frederico Castelo Moura ${ }^{2}$

Mário Luiz Ribeiro Monteiro ${ }^{3}$

\section{RESUMO}

Objetivo: Comparar a capacidade do polarímetro de varredura a laser(GDx), do tomógrafo de coerência óptica(OCT) 1 e do Stratus-OCT em diferenciar olhos normais de olhos com atrofia em banda do nervo óptico e hemianopsia temporal. Métodos: Vinte e três olhos de pacientes com atrofia em banda do nervo óptico e 23 olhos de indivíduos normais foram incluídos em estudo prospectivo observacional caso-controle. Todos foram submetidos à análise da camada de fibras nervosas retiniana (CFNR) utilizando GDx, OCT-1 e Stratus-OCT. As médias dos valores obtidos em cada aparelho foram comparadas entre olhos com atrofia em banda e controles normais. Curvas ROC (receiver operating characteristic) e sensibilidade para especificidades fixas $(80 \%$ e $95 \%$ ) foram calculadas para cada parâmetro produzido pelos três instrumentos e comparadas entre si. Resultados: Quando comparados aos indivíduos normais, os resultados dos pacientes referentes à camada de fibras nervosas retiniana (média global e quatro quadrantes estudados) foram significativamente menores $(p<0,05)$ em todos os aparelhos estudados, com exceção do parâmetro referente ao setor temporal quando avaliado pelo GDx. A comparação das áreas sob a curva ROC (AROC) dos parâmetros referentes aos três aparelhos mostrou valores significativamente maiores para o Stratus-OCT quando comparados ao OCT-1 na média global e no setor temporal. O Stratus-OCT foi significativamente mais sensível do que o GDx na média global e nos quadrantes temporal, nasal e inferior. Por sua vez, o OCT-1 foi superior ao GDx na discriminação dos defeitos apenas no quadrante temporal, não mostrando diferença significativa na média global e nos demais quadrantes. Os três aparelhos mostraram capacidade semelhante na identificação dos defeitos referentes ao quadrante superior. Conclusão: O Stratus-OCT demonstrou a maior capacidade em diferenciar olhos com atrofia em banda do nervo óptico de olhos normais embora todos os três aparelhos consigam identificar a maioria dos olhos anormais. O OCT-1 se mostrou inferior ao Stratus-OCT e superior ao GDx no quadrante temporal. Embora o GDx tenha sido capaz de identificar algum tipo de alteração na maioria dos olhos estudados, este aparelho se mostrou pouco sensível na detecção da perda axonal nos quadrantes nasal e temporal do disco óptico, devendo ser utilizado com cuidado no estudo de afecções neuroftalmológicas que acometem estas regiões do disco óptico.

Descritores: Tomografia de coerência óptica; Nervo óptico; Retina/patologia; Perimetria; Lasers/uso diagnóstico; Fibras nervosas; Atrofia óptica; Microscopia confocal; Quiasma óptico; Axônios/patologia; Técnicas de diagnóstico oftalmológico; Sensibilidade e especificidade; Estudo comparativo 


\section{INTRODUCÃO}

A compressão quiasmática por tumores supra-selares é uma das afecções mais comuns da via óptica que caracteristicamente leva a hemianopsia bitemporal por compressão das fibras nervosas que cruzam no quiasma óptico ${ }^{(1)}$ e atrofia óptica. O padrão de perda da camada de fibras nervosas retiniana (CFNR) nos pacientes com compressão quiasmática é também um elemento diagnóstico importante. Pacientes com lesões medianas do quiasma que afetam as fibras cruzadas e que mostram defeito campimétrico temporal com preservação do hemicampo nasal demonstram perda das fibras cruzadas com preservação das não cruzadas que se originam na hemiretina temporal e penetram o nervo óptico pelos feixes de fibras temporais superior e inferior. Por conseguinte a perda da CFNR ocorre predominantemente nos setores nasal e temporal do disco óptico, um padrão identificado à oftalmoscopia como atrofia em banda $(\mathrm{AB})$ do nervo óptico que é importante no diagnóstico e seguimento dos pacientes com compressão quiasmática $^{(2)}$. Até recentemente, o exame da CFNR nos olhos com $\mathrm{AB}$ se realizava de forma subjetiva e qualitativa. Com o surgimento dos métodos ópticos de imagem, como o tomógrafo de coerência óptica (OCT) e o polarímetro de varredura a laser (GDx), o exame vem sendo feito também de forma objetiva e quantitativa.

A habilidade do OCT e do GDx de avaliar a CFNR já foi comprovada por vários trabalhos utilizando pacientes com glaucoma ${ }^{(3-4)}$, e outras afecções do nervo óptico ${ }^{(5-7)}$, mas poucos são os estudos avaliando tais aparelhos nas afecções quiasmáticas. Alguns autores utilizaram o GDx para quantificar a CFNR dos olhos com AB do nervo óptico ${ }^{(8)}$. Demonstrou-se que o GDx identificou como anormais todos os olhos estudados e foi capaz de detectar a perda axonal que ocorreu nas regiões superior, inferior e nasal, falhando, no entanto na identificação da perda axonal no setor temporal. Neste estudo não foi verificada a sensibilidade e a especificidade de cada parâmetro. Dois estudos prévios ${ }^{(9-10)}$ utilizando o OCT-1 (primeira versão disponível comercialmente do OCT) apresentaram resultados promissores, pois o instrumento permitiu diferenciar a anormalidade em cada um dos quadrantes dos olhos com AB separando-os dos olhos normais. Observou-se, no entanto, grande superposição com valores normais. Além disso, a identificação como sendo anormal em um determinado indivíduo se mostra difícil pelo OCT-1, já que o aparelho não apresenta uma faixa com os limites da normalidade, não possuindo a mesma facilidade de utilização do GDx. Recentemente, o novo Stratus-OCT (Carl Zeiss Meditec, Inc, Dublin, CA) foi introduzido com várias melhorias sobre o OCT-1. Apresenta melhor resolução devido a um número maior de scans $\mathrm{A}$ e um tempo de aquisição mais rápido e, a exemplo do GDx, fornece informação da probabilidade de anormalidade quando comparado com um banco de dados interno pré-estabelecido. Nenhum estudo prévio utilizou o Stratus OCT em pacientes com afecção quiasmática ou comparou diretamente o GDx com o OCT nos pacientes com AB do nervo óptico.
O objetivo desse trabalho foi, portanto, comparar num mesmo grupo de olhos, a habilidade do GDx, do OCT-1 e do Stratus-OCT de diferenciar olhos normais de olhos com atrofia em banda do nervo óptico e hemianopsia temporal e verificar a sensibilidade e a especificidade de cada um destes aparelhos.

\section{MÉTODOS}

Entre dezembro de 2002 e dezembro de 2003, foram examinados em um estudo prospectivo observacional caso-controle, 46 olhos de 37 indivíduos no serviço de neuro-oftalmologia do Hospital das Clínicas da Faculdade de Medicina da Universidade de São Paulo. Vinte e três olhos foram de 19 pacientes portadores de tumores supraselares já submetidos a tratamento clínico e/ou cirúrgico e com evidências neuro-radiológicas de compressão pregressa (já tratados) da via óptica e defeito campimétrico seqüelar em hemicampo temporal. Quinze pacientes eram portadores de macroadenoma hipofisário, três de craniofaringioma e um de meningioma supra-selar. Os 23 olhos restantes foram de 18 indivíduos do grupo controle normal, emparelhados por sexo e idade ao grupo de estudo. O protocolo da pesquisa foi aprovado pela comissão de ética da nossa instituição.

O exame de campo visual (CV) foi realizado com o perímetro de Goldmann, usando-se os estímulos V-4-e, I-4-e, I-3-e, I-2-e e I-1-e para desenhar as isópteras. Os pacientes também foram submetidos ao perímetro computadorizado (Humphrey, 24-2 "Full-Threshold test").

Os pacientes obedeceram aos seguintes critérios de inclusão: acuidade visual corrigida melhor que $20 / 30$ no olho a ser estudado; erro de refração menor que 6 dioptrias esféricas e 3 dioptrias cilíndricas; pressão intra-ocular menor que $22 \mathrm{mmHg}$; hemianopsia temporal completa ao perímetro de Humphrey e hemianopsia temporal completa ou quase completa ao perímetro de Goldmann; hemicampo nasal dentro dos limites normais; defeito perimétrico estável há pelo menos 1 ano após tratamento clínico ou cirúrgico do tumor; estudo neuro-radiológico recente demonstrando ausência de compressão atual da via óptica; ausência de afecções oculares e de história de enxaqueca clássica e boa colaboração para realização dos exames. Um CV de Humphrey confiável foi definido como aquele com menos do que $25 \%$ de perda de fixação, de respostas falso-negativas e de respostas falso-positivas.

No grupo controle foram incluídos pacientes da triagem, acompanhantes ou funcionários do ambulatório de oftalmologia do Hospital das Clínicas da Universidade de São Paulo que obedeciam aos seguintes critérios: olho e idade emparelhados com o respectivo paciente do grupo em estudo, aceitando-se um desvio de mais ou menos cinco anos; acuidade visual corrigida melhor que 20/30, com erro de refração menor que seis dioptrias esféricas e três dioptrias cilíndricas; ausência de defeito campimétrico avaliado pela perimetria computadorizada (Humphrey, 24-2 SITA Standard test); ausência de afecções oculares ou história de crises de enxaqueca clássica e boa colaboração para realização dos exames. 
Todos os pacientes foram submetidos a exame oftalmológico completo e à realização de perimetria Humphrey (Humphrey systems, San Diego, CA, modelo HFA II 750), polarimetria a laser (AFN/GDx); tomografia de coerência óptica (OCT) 1, versão A5 e Stratus OCT versão 3.0, Carl Zeiss Meditec Inc, Dublin, CA). Os exames de CV e OCT foram realizados no mesmo dia ou dentro de um intervalo de tempo máximo de 2 semanas.

Para realização do GDx, os pacientes foram submetidos, por um mesmo examinador, a uma sessão de seis imagens de cada olho acometido com o GDX (compensador corneano fixo), e uma imagem média (mean) foi criada a partir das três melhores imagens. O desvio padrão (DP) da imagem média foi menor ou igual a oito micra para inclusão. Avaliamos a espessura da CFNR média (average) nasal, temporal, superior e inferior. Para o OCT e Stratus OCT, os pacientes foram submetidos a uma sessão para obtenção de cinco imagens. Para adquirir a imagem, utiliza-se um scan circular ao redor do centro do disco óptico utilizando-se um círculo padrão de 3,4 $\mathrm{mm}$ de diâmetro disponível no software do aparelho, de tal forma a permitir a medida da CFNR peripapilar. No OCT-1 e no Stratus-OCT, foram avaliadas as espessuras da CFNR nos quadrantes nasal,temporal, superior, inferior, na média de 360 graus (average) e em cada um de 12 segmentos de 30 graus, identificados como "horas de relógio", numerados de 1 a 12 .

Após realização dos exames, os pacientes de ambos os grupos tiveram suas imagens elegíveis obtidas pelo GDx, OCT-1 e Stratus-OCT comparadas e avaliadas estatisticamente com o respectivo grupo controle pelo teste $t$ de Student. Utilizaram-se curvas ROC (receiver operating characteristic) para avaliar o poder discriminativo de cada parâmetro dos três instrumentos para diferenciar olhos com $\mathrm{AB}$ de olhos normais. A curva ROC mostra a relação entre os valores de sensibilidade e 1-especificidade de um teste diagnóstico. Na curva ROC, o eixo Y mostra a sensibilidade do teste e o eixo X mostra a taxa de erro falso-positivo (1-especificidade). A área sob a curva ROC (AROC) pode ser interpretada como o valor médio de sensibilidade para todos os valores possíveis de especificidade ou, de maneira análoga, o valor médio de sensibilidade para todos os valores possíveis de especificidade ou, de maneira análoga, o valor médio de especificidade para todos os valores possíveis de sensibilidade ${ }^{(11-12)}$. Uma AROC de 1,0 representa um poder discriminativo perfeito para distinguir olhos com $\mathrm{AB}$ de olhos normais, enquanto que uma $\mathrm{AROC}$ de 0,5 representa um poder discriminativo nulo. As AROCs de cada parâmetro foram calculadas e comparadas entre si usando o método não paramétrico proposto por alguns autores ${ }^{(13)}$.

Valor de P menor que 0,05 foi considerado estatisticamente significante. Embora a realização de múltiplos testes de hipótese envolvendo a mesma amostra, possa levar a aumento na chance de erro tipo I, optamos por não aplicar métodos de correção para múltiplas comparações, uma vez que estes aumentam a chance de erro tipo II. As análises estatísticas foram feitas usando os pacotes estatísticos SPSS versão 11,0 (SPSS inc, Chicago, Ill) e NCSS versão 2004 (NCSS inc., Kaysville, Utah).

RESULTADOS

Um total de 37 indivíduos, 18 normais e 19 pacientes com atrofia em banda do nervo óptico, foram incluídos no estudo com um total de 46 olhos avaliados ( 23 olhos em cada grupo). A média de idade no grupo com atrofia em banda foi de 46,65 \pm 14,80 anos sem diferença significativa para o grupo controle, $46,1 \pm 13,58$ anos. A distribuição de sexo e raça foi equivalente pelo pareamento entre os grupos, com predomínio de homens brancos, sendo 16 olhos $(69,56 \%)$ de indivíduos do sexo masculino e 7 olhos $(30,43 \%)$ de indivíduos do sexo feminino. Foram incluídos no estudo, 4 olhos de indivíduos negros em cada grupo. Os exames de CV computadorizado foram realizados nos dois grupos; nos 23 olhos com atrofia, o valor médio de MD foi de $-15,03 \pm 2,13$ e nos 23 olhos normais o MD foi de $-1,29 \pm 0,85$ mostrando diferença significativa entre os dois grupos $(\mathrm{p}<0,001)$. Fato semelhante ocorreu com o valor médio do PSD, que no grupo da atrofia foi de $14,85 \pm 1,83$ e nos controles de 1,43 $\pm 0,19(\mathrm{p}<0,001)$. Todos os pacientes foram submetidos ao OCT-1 e ao Stratus OCT conforme descrito anteriormente e os dados dos parâmetros analisados (quadrantes nasal, temporal, superior, inferior, average e segmentos de $30^{\circ}$ da elipse) foram comparados entre os dois grupos, considerando-se o valor de $\mathrm{p}<0,05$ como estatisticamente significativo (Tabelas 1 a 3 ). Todos os valores mostraram diferenças estatisticamente significativas para o OCT-1 e Stratus OCT e apenas no setor temporal do GDx essa diferença não foi observada entre olhos normais e olhos com atrofia em banda do nervo óptico (Tabelas 1 a 3 ).

Os valores (em micra) da espessura da CFNR para cada parâmetro avaliado pelo Stratus OCT, OCT-1 e GDx foram calculados e comparados (valores de p). A área sob a curva ROC e suas sensibilidades em especificidades fixas ( $\geq 95 \%$ e $\geq 80 \%$ ) também foram calculadas e comparadas. Os dados obtidos foram demonstrados nas tabelas 1 a 3 .

Utilizando-se o método de DeLong e col. ${ }^{(13)}$ foi feita a comparação entre as AROCs referente aos valores da média global e de cada um dos quatro quadrantes para os três. Com relação à média global a AROC obtida com o Stratus-OCT foi 0,999 , significativamente maior $(\mathrm{p}=0,048)$ que aquela referente ao OCT- 1 cujo valor foi de 0,955 , e também significativamente maior ( $\mathrm{p}=0,018$ ) que aquela referente ao GDx, de 0,879. Não houve diferença estatisticamente significante entre a AROC para a média global referente ao OCT-1 e o GDx $(\mathrm{p}=0,123)$.

Não houve diferença estatisticamente significante entre as AROCs referentes ao quadrante superior obtidas com o Stratus-OCT $(0,984)$, OCT-1 $(0,910)$ e GDx $(0,864)$ mostrando que os três aparelhos foram igualmente sensíveis para detectar anormalidades neste quadrante. No quadrante inferior não houve diferença estatisticamente significante entre a AROC referente ao $\operatorname{GDx}(0,889)$ e o OCT $-1(0,965)$ sendo o $\mathrm{p}=0,143 \mathrm{e}$ nem entre o OCT-1 e o Stratus-OCT $(0,993)$, sendo o $\mathrm{p}=0,062$. Observou-se, no entanto, diferença significativa entre o Stratus-OCT e o GDx neste quadrante $(\mathrm{p}=0,012)$. 
534 Comparação entre o polarímetro de varredura a laser, a tomografia de coerência óptica 1 e o Stratus-OCT na detecção da perda axonal da atrofia em banda do nervo óptico

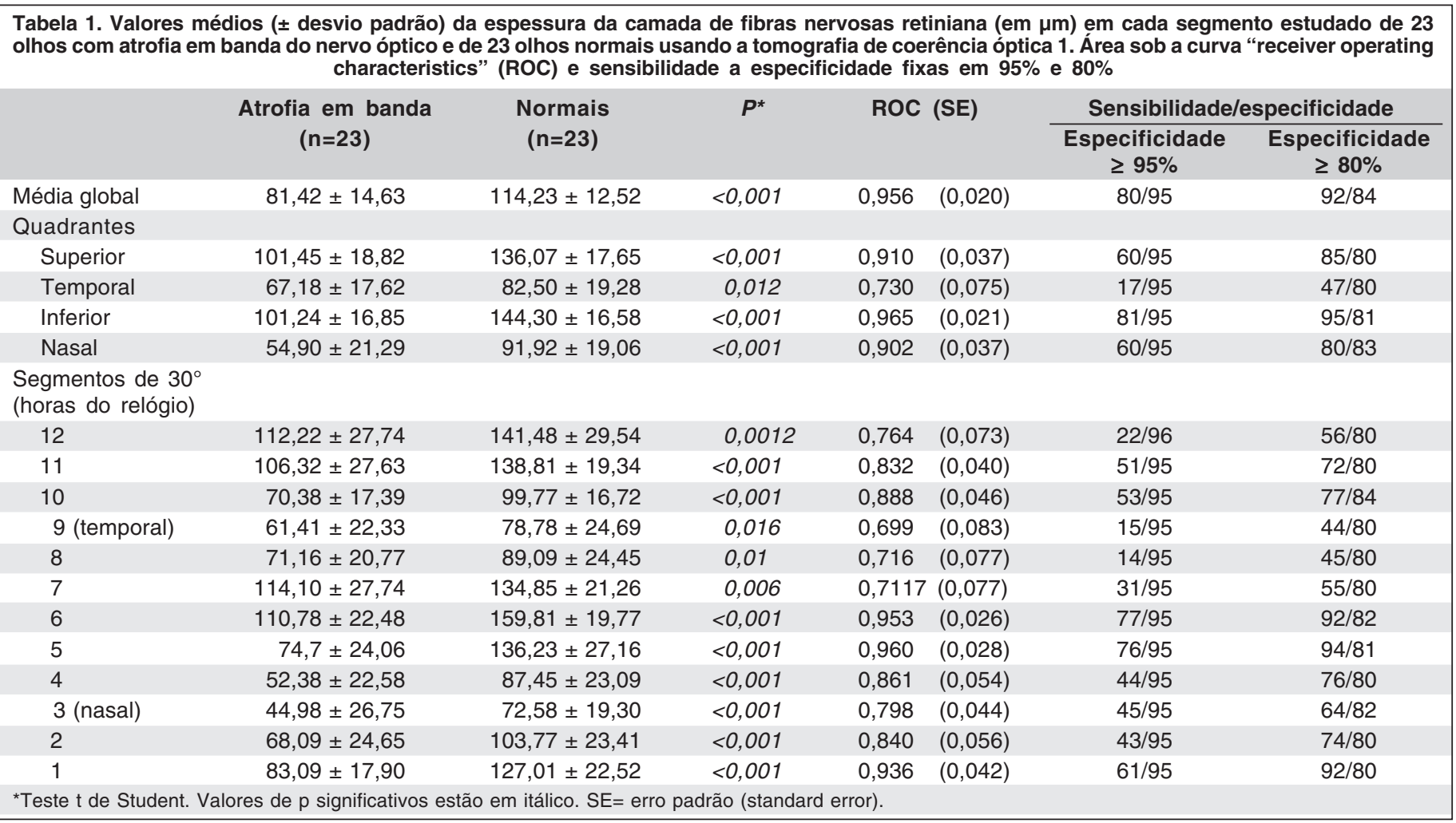

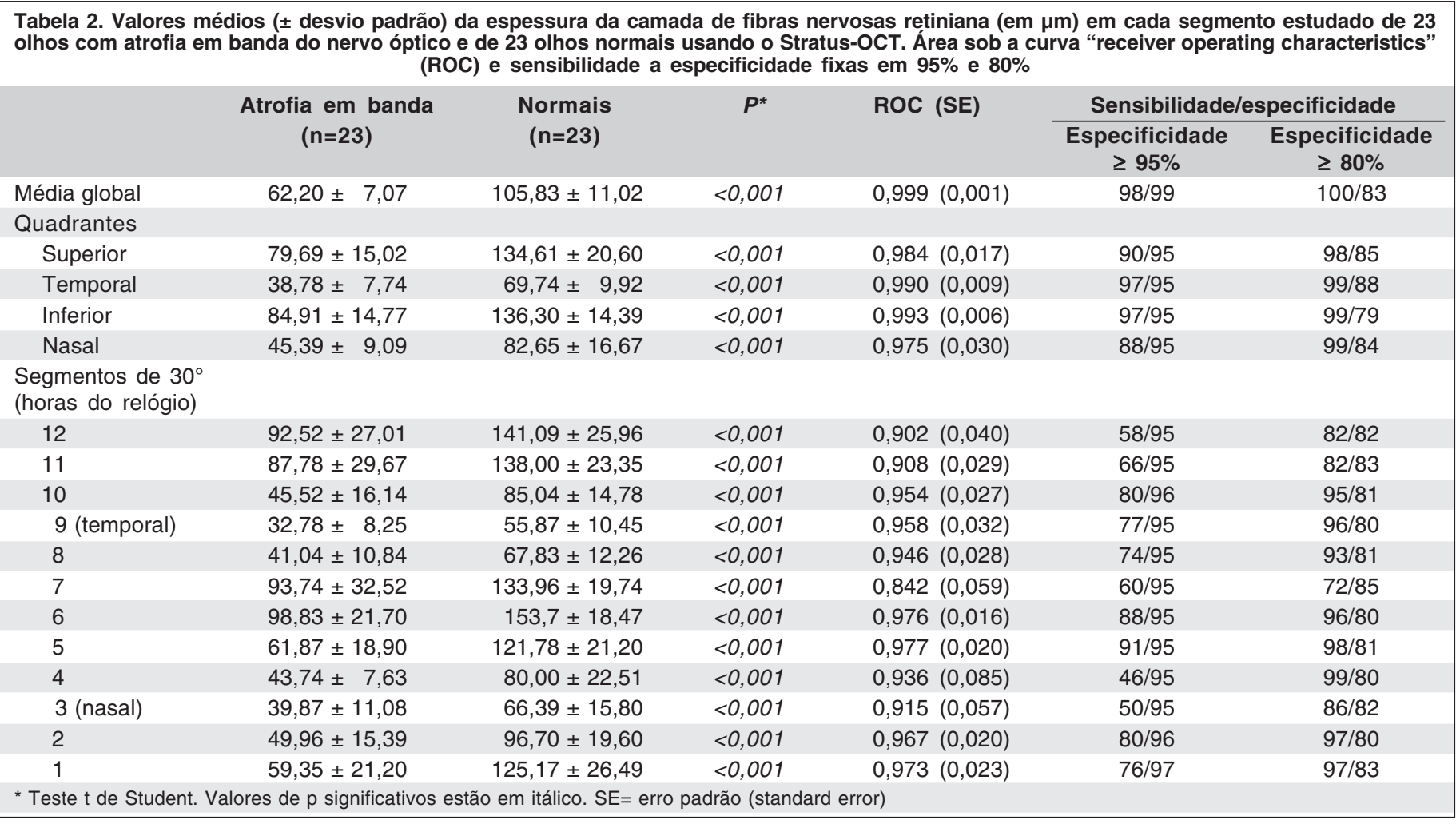

Diferença maior foi observada na análise dos parâmetros referentes aos quadrantes nasal e temporal do disco óptico, justamente aqueles mais diretamente acometidos nos olhos com atrofia em banda do nervo óptico. No setor temporal, a 


\begin{tabular}{|c|c|c|c|c|c|c|}
\hline \multirow[b]{3}{*}{ Média global } & \multirow{2}{*}{$\begin{array}{l}\text { Atrofia em banda } \\
\qquad(n=23)\end{array}$} & \multirow{2}{*}{$\begin{array}{c}\text { Normais } \\
(n=23)\end{array}$} & \multirow[t]{2}{*}{$P^{*}$} & \multirow[t]{2}{*}{ ROC (SE) } & \multicolumn{2}{|c|}{ Sensibilidade/especificidade } \\
\hline & & & & & $\begin{array}{c}\text { Especificidade } \\
\geq 95 \%\end{array}$ & $\begin{array}{l}\text { Especificidade } \\
\quad \geq 80 \%\end{array}$ \\
\hline & $48,78 \pm 9,29$ & $64,03 \pm 9,12$ & $<0,001$ & $0,879(0,048)$ & $47 / 96$ & $79 / 80$ \\
\hline \multicolumn{7}{|l|}{ Quadrantes } \\
\hline Superior & $49,79 \pm 11,70$ & $68,99 \pm 12,90$ & $<0,001$ & $0,865(0,058)$ & $43 / 95$ & $76 / 80$ \\
\hline Temporal & $38,63 \pm 8,39$ & $40,08 \pm 10,01$ & 0,597 & $0,520(0,088)$ & $6 / 92$ & $20 / 80$ \\
\hline Inferior & $56,19 \pm 11,41$ & $78,00 \pm 13,68$ & $<0,001$ & $0,889(0,057)$ & $47 / 95$ & $79 / 82$ \\
\hline Nasal & $40,96 \pm 10,13$ & $48,37 \pm 9,04$ & 0,012 & $0,707(0,065)$ & $23 / 95$ & $49 / 80$ \\
\hline
\end{tabular}

AROC obtida com o GDx foi de apenas 0,544, a do OCT-1 subiu para 0,721 e a do Stratus-OCT foi de 0,993 . O estudo estatístico mostrou diferença significativa quando comparamos o Stratus-OCT tanto com o GDx ( $\mathrm{p}=0,001)$ como com o OCT- 1 $(\mathrm{p}=0,003)$. Não houve, no entanto, diferença significativa na comparação entre o GDx e o OCT-1 ( $\mathrm{p}=0,197)$. Quanto ao setor nasal a AROC foi de 0,707 para o GDx, 0,902 para o OCT-1 e 0,975 para o Stratus-OCT. Houve diferença estatisticamente significativa na comparação entre o GDx e o OCT-1 $(\mathrm{p}=0,014)$ e entre o GDx e o Stratus-OCT ( $\mathrm{p}=0,034$ ), não se observando diferença significativa entre o OCT-1 e o Stratus-OCT ( $\mathrm{p}=0,248)$.

Comparamos também as áreas sob a curva ROC referentes aos segmentos de 30 graus estudados com o OCT-1 e o Stratus-OCT. Observamos que as AROCs referentes ao StratusOCT foram quase sempre maiores do que aquelas referentes ao OCT-1 (Tabela 4). Significância estatística foi observada, no entanto, apenas na comparação entre os dois equipamentos no que se referiu às medidas dos segmentos de 30 graus correspondentes aos meridianos das 9, 8, 7 horas (temporais).

\begin{tabular}{|c|c|c|c|}
\hline \multicolumn{4}{|c|}{$\begin{array}{l}\text { Tabela 4. Comparação, usando o método de DeLong et al., entre as } \\
\text { áreas sob a curva Receiver Operating Characteristics (AROCs) } \\
\text { referentes a cada um dos segmentos de } 30 \text { graus correspondentes } \\
\text { às horas do relógio, obtidas com o OCT-1 e o Stratus-OCT }{ }^{(13)}\end{array}$} \\
\hline $\begin{array}{l}\text { Segmentos de } 30^{\circ} \\
\text { (horas do relógio) }\end{array}$ & $\begin{array}{l}\text { AROC } \\
\text { OCT-1 }\end{array}$ & $\begin{array}{c}\text { AROC } \\
\text { Stratus-OCT }\end{array}$ & $P^{*}$ \\
\hline 12 & 0,764 & 0,902 & 0,059 \\
\hline 11 & 0,832 & 0,908 & 0,068 \\
\hline 10 & 0,885 & 0,964 & 0,095 \\
\hline 9 (temporal) & 0,699 & 0,958 & 0,019 \\
\hline 8 & 0,711 & 0,949 & 0,006 \\
\hline 7 & 0,723 & 0,854 & 0,046 \\
\hline 6 & 0,949 & 0,972 & 0,260 \\
\hline 5 & 0,960 & 0,977 & 0,394 \\
\hline 4 & 0,867 & 0,937 & 0,227 \\
\hline 3 (nasal) & 0,798 & 0,915 & 0,186 \\
\hline 2 & 0,853 & 0,969 & 0,068 \\
\hline 1 & 0,936 & 0,973 & 0,419 \\
\hline
\end{tabular}

\section{DISCUSSÃO}

Este é o primeiro estudo comparativo da acurácia diagnóstica do OCT-1, do Stratus OCT e do GDx feito na mesma amostra populacional de pacientes com atrofia em banda do nervo óptico. Olhos com este tipo de atrofia representam um modelo importante de estudo da acurácia dos aparelhos de medição da CFNR, especialmente nos setores temporal e nasal do disco óptico, que são particularmente afetados neste tipo de $\operatorname{atrofia}^{(1-2)}$.

Recentemente, iniciamos uma linha de pesquisa utilizando o GDx e a tomografia de coerência óptica, analisando quantitativamente a CFNR dos olhos com atrofia em banda do nervo óptico e verificando a capacidade do instrumento de diferenciar esses olhos de olhos controles normais ${ }^{(8-9)}$. Todos os 23 olhos estudados seguiam um padrão bastante específico de perda da camada de fibras nervosas, com hemianopsia temporal grave e preservação do hemicampo nasal e de boa acuidade visual. No estudo atual, mantivemos os mesmos critérios de seleção dos olhos estudados e ampliamos a amostra populacional, acrescentamos a mensuração da CFNR através do Stratus-OCT. Aprofundamos também a análise comparativa do poder diagnóstico de cada um dos aparelhos com a realização da comparação das áreas sob a curva ROC (AROC's) e das sensibilidades sob especificidades fixas de cada parâmetro fornecido pelos instrumentos analisados.

Nosso estudo demonstrou que tanto o GDx como o OCT-1 e o Stratus-OCT foram capazes de diferenciar olhos normais de olhos com AB. No entanto, confirmamos nossa observação anterior de que o GDx não foi capaz de identificar alterações na CFNR no setor temporal. Encontramos diferenças estatisticamente significativas para todos os parâmetros estudados na comparação dos pacientes com o grupo controle, à exceção do parâmetro setor temporal do GDx $(\mathrm{p}=0,597)$. Esse dado mostrou concordância com estudo prévio( ${ }^{(8)}$ que também não evidenciou diferença estatisticamente significativa para o mesmo parâmetro. Com isso, queremos alertar que o GDx pode não detectar uma perda axonal no setor temporal. Embora o GDx tenha considerado anormal a maioria dos olhos com $\mathrm{AB}$, este 
aparelho mostrou baixa sensibilidade nos setores temporal e nasal do disco óptico, indicando uma limitação importante em sua utilização em afecções neuroftalmológicas que muitas vezes acometem estas regiões. O fato de ter identificado como anormal os valores médios da maioria dos pacientes, provavelmente se deveu ao fato de nossa amostra ter incluído indivíduos com defeitos campimétricos importantes, com hemianopsia temporal completa ou quase completa.

Trabalhos prévios já mostraram que a compensação corneana fixa da primeira versão do GDx promovia alteração na medida da $\mathrm{CFNR}^{(14)}$, mais especificamente nos setores nasal e temporal do disco óptico. Em decorrência disso, os fabricantes desenvolveram um compensador corneano variável para a versão mais recente (GDx VCC), aumentando a acurácia diagnóstica do método ${ }^{(17)}$. Em recente estudo, alguns autores verificaram a capacidade da tomografia por coerência óptica sensível à polarização (OS-OCT), um método experimental, em fornecer informações objetivas sobre a espessura da CFNR e sua birrefringência ${ }^{(18)}$. Os autores observaram que as medidas de birrefringência variaram dentro de um mesmo círculo peripapilar de medida, gerando valores de espessura maiores nas porções superiores e inferiores do disco óptico, mostrando que, com a utilização de birrefringência espacial fixa, a conversão do scan de polarimetria a laser em espessura da CFNR poderá apresentar valores incorretos. No estudo de Huang et al., fato semelhante também foi observado ${ }^{(19)}$. Nos estudos com $\mathrm{AB}^{(8,10)}$ também foi considerado que o GDx com compensação corneana variável poderia melhorar a análise da CFNR nos setores nasal e temporal. Embora não existam ainda publicações avaliando olhos com AB usando o GDx VCC, estudos preliminares realizados no nosso serviço, mostraram, no entanto, baixa acurácia também do GDx VCC na detecção da perda axonal horizontal existente na $\mathrm{AB}$, indicando que a falha na detecção da perda axonal no quadrante temporal do nervo óptico parece não depender do compensador fixo de córnea do aparelho antigo e sim de uma limitação outra do aparelho no que se refere à mensuração destes setores. No entanto, estudos adicionais são necessários para confirmar esse resultado.

Nosso estudo demonstrou que tanto o OCT-1 como o Stratus-OCT foram capazes de diferenciar olhos normais de olhos com AB, no parâmetro "média global", assim como nos quatro quadrantes estudados e em cada um dos 12 segmentos de 30 graus. Observamos, no entanto, que as diferenças entre os olhos normais foram mais acentuadas com o Stratus-OCT do que com o OCT-1. Para melhor comparar a sensibilidade e a especificidade de cada um destes aparelhos, estudamos as AROCs de cada um deles e comparamos os valores entre si. Estudos semelhantes já foram realizados em pacientes com glaucoma. Zangwill et al., comparando OCT 2000 e GDx em pacientes com glaucoma não encontrou diferença significativa entre as AROC's dos melhores parâmetros obtidos, entretanto o melhor parâmetro do OCT 2000 demonstrou maior sensibilidade do que o melhor parâmetro do $\mathrm{GDx}^{(20)}$. Medeiros et al., em estudo com pacientes glaucomatosos, encontraram
AROC's sem diferença significante entre os melhores parâmetros do GDx VCC e Stratus-OCT ${ }^{(21)}$.

Nosso estudo é, no entanto, o primeiro a realizar a comparação entre a sensibilidade e a especificidade de cada um dos aparelhos em pacientes com AB, na qual o padrão de perda da CFNR é bastante diferente daquele observado em indivíduos com glaucoma. A comparação dos instrumentos demonstrou que o OCT e o Stratus-OCT possuem uma capacidade superior à do GDx em identificar a perda axonal da $\mathrm{AB}$, com uma superioridade do Stratus-OCT em relação ao OCT-1, provavelmente, devido a melhor resolução do Stratus OCT ${ }^{(22)}$. A comparação entre as áreas sob as curvas ROC mostrou claramente a superioridade do Stratus-OCT sobre o OCT-1 e o GDx (e também do OCT-1 sobre o GDx). Observamos que apenas no quadrante superior não houve diferença significativa entre os três aparelhos. Em todas as demais mensurações (média global, quadrante temporal, inferior e nasal) o Stratus-OCT foi superior ao GDx. Acreditamos que a superioridade do OCT sobre o GDx possa ser facilmente explicada pela dificuldade que o polarímetro a laser tem em analisar os setores nasal e temporal do disco óptico como já observado por outros autores. Por outro lado, como o Stratus-OCT representa uma evolução sobre as versões iniciais do OCT, com uma aquisição mais rápida das imagens e com um maior número de scans por segmento estudado. A observação de sua melhor resolução e melhor poder de discriminação confirmou a expectativa que tínhamos antes do início do estudo.

\section{CONCLUSÃO}

Em conclusão do estudo atual mostramos que todos os três aparelhos foram capazes de identificar olhos com atrofia em banda apresentando grave defeito campimétrico temporal, embora o GDx tenha deixado de identificar a perda da CFNR no setor temporal. O estudo atual evidenciou que o Stratus-OCT demonstrou a maior capacidade em diferenciar olhos com atrofia em banda do nervo óptico de olhos normais. O OCT-1 se mostrou inferior ao Stratus-OCT e superior ao GDx no quadrante temporal. Embora o GDx tenha sido capaz de identificar anormalidade na maioria dos olhos estudados, este aparelho se mostrou pouco sensível na detecção da perda axonal nos quadrantes nasal e temporal do disco óptico, devendo ser utilizado com cuidado no estudo de afecções neuroftalmológicas que acometem estas regiões do disco óptico.

\section{ABSTRACT}

Purpose: To compare the abilitiy of scanning laser polarimetry (GDx), optical coherence tomography (OCT) 1 and Stratusoptical coherence tomography to discriminate between healthy eyes and eyes with band atrophy of the optic nerve and temporal hemianopsia. Methods: Twenty-three eyes with band atrophy of the optic nerve and 23 eyes from healthy subjects 
were included in this observational prospective case-control study. All eyes underwent retinal nerve fiber layer (RNFL) thickness analysis using GDx, optical coherence tomography-1 and Stratus-optical coherence tomography. Mean values obtained with each equipment were compared between band atrophy and normal eyes. Receiver operating characteristic (ROC) curves and sensitivities at fixed specificities (80\% e 95\%) were calculated for each parameter calculated with each equipment and compared. Results: When compared with healthy subjects, the parameters corresponding to the global average as well as each of the four quadrant retinal nerve fiber layer thickness in eyes with band atrophy were significantly smaller $(\mathrm{p}<0.05)$, than in normal eyes, with the exception of GDx's temporal thickness parameter. Comparison of the areas under ROC curves (AUCs) of the parameters from the three equipments revealed significantly greater values for the Stratus-OCT when compared to the OCT-1 in the global average and in the temporal quadrant thickness measurement. Stratus-OCT was significantly more sensitive than GDx in the global average as well as in the temporal, nasal and inferior quadrant. OCT-1 was superior to GDx only in the temporal quadrant. All three equipments revealed a similar ability to identify retinal nerve fiber layer reduction in the superior quadrant. Conclusions: The Stratus OCT showed the best ability to discriminate between eyes with band atrophy of the optic nerve and healthy eyes although all three equipments were able do identify most of the abnormal eyes. OCT-1 was inferior to Stratus-OCT and superior do GDx in the temporal quadrant. Although GDx was able identify some abnormality in most studied eyes, the equipment showed poor sensitivity in the detection of axonal loss in the nasal and temporal aspect of the optic disc and should be used with caution in neuro-ophthalmic diseases that cause retinal nerve fiber layer loss in those areas of the optic disc.

Keywords: Tomography, optical coherence; Optic nerve; Retina/pathology; Perimetry; Lasers/diagnostic use; Nerve fibers; Optic atrophy; Microscopy, confocal; Optic chiasm; Axons/ pathology; Diagnostic techniques, ophthalmological; Sensitivity and specificity; Comparative study

\section{REFERÊNCIAS}

1. Unsold R, Hoyt WF. Band atrophy of the optic nerve. The histology of temporal hemianopsia. Arch Ophthalmol. 1980;98(9):1637-8.

2. Mikelberg FS, Yidegiligne HM. Axonal loss in band atrophy of the optic nerve in craniopharyngioma: a quantitative analysis. Can J Ophthalmol. 1993;28(2): 69-71.

3. Guedes V, Schuman JS, Hertzmark E, Wollstein G, Correnti A, Mancini R., et al. Optical coherence tomography measurement of macular and nerve fiber layer thickness in normal and glaucomatous human eyes. Ophthalmology. 2003;110 (1):177-89.

4. Medeiros FA, Susanna Junior, R. Análise de Fourier para detecção de defeitos localizados na camada de fibras nervosas da retina com a polarimetria a laser. Arq Bras Oftalmol. 2003;66(6):846-53.

5. Steel DH, Waldock A. Measurement of the retinal nerve fibre layer with scanning laser polarimetry in patients with previous demyelinating optic neuritis. J Neurol Neurosurg Psychiatry. 1998;64(4):505-9.

6. Medeiros FA, Moura FC, Vessani RM, Susanna R Jr. Axonal loss after traumatic optic neuropathy documented by optical coherence tomography. Am J Ophthalmol. 2003;135(3):406-8.

7. Zoumalan CI, Agarwal M, Sadun AA. Optical coherence tomography can measure axonal loss in patients with ethambutol-induced optic neuropathy. Graefes Arch Clin Exp Ophthalmol. 2005;243(5):410-6.

8. Monteiro ML, Medeiros FA, Ostroscki MR. Quantitative analysis of axonal loss in band atrophy of the optic nerve using scanning laser polarimetry. Br J Ophthalmol. 2003;87(1):32-7.

9. Monteiro ML, Leal BC, Rosa AA, Bronstein MD. Optical coherence tomography analysis of axonal loss in band atrophy of the optic nerve. $\mathrm{Br} \mathrm{J}$ Ophthalmol. 2004;88(7):896-9.

10. Kanamori A, Nakamura M, Matsui N, Nagai A, Nakanishi Y, Kusuhara S, et al. Optical coherence tomography detects characteristic retinal nerve fiber layer thickness corresponding to band atrophy of the optic discs. Ophthalmology. 2004;111(12):2278-83. Comment in: Ophthalmology. 2005;112(11):2055-6; author reply 2056-7.

11. Martinez EZ, Louzada-Neto F, Pereira BB. A curva ROC para testes diagnósticos. Cad Saúde Colet (Rio J.). 2003;11(1):7-31.

12. Zhou X-H, Obuchowski N, Mcclish D. Statistical Methods in Diagnostic Medicine. In. New York: John Wiley \& Sons, Inc., 2002; v. Cap 4.

13. DeLong ER, DeLong DM, Clarke-Pearson DL. Comparing the areas under two or more correlated receiver operating characteristic curves: a nonparametric approach. Biometrics. 1988;44(3):837-45.

14. Greenfield DS, Knighton RW, Huang XR. Effect of corneal polarization axis on assessment of retinal nerve fiber layer thickness by scanning laser polarimetry. Am J Ophthalmol. 2000;129(6):715-22. Comment in: Am J Ophthalmol. 2001;131(4):528-9; Am J Ophthalmol. 2001;131(3):403-4.

15. Morgan JE, Waldock A, Jeffery G, Cowey A. Retinal nerve fibre layer polarimetry: histological and clinical comparison. Br J Ophthalmol. 1998;82(6):684-90.

16. Kogure S, Chiba T, Kinoshita T, Kowa H, Tsukahara S. Effects of artefacts on scanning laser polarimetry of retinal nerve fibre layer thickness measurement. $\mathrm{Br}$ J Ophthalmol. 2000;84(9):1013-7.

17. Weinreb RN, Bowd C, Zangwill LM. Scanning laser polarimetry in monkey eyes using variable corneal polarization compensation. J Glaucoma. 2002;11(5): 378-84.

18. Cense B, Chen TC, Park BH, Pierce MC, de Boer JF. Thickness and birefringence of healthy retinal nerve fiber layer tissue measured with polarization-sensitive optical coherence tomography. Invest Ophthalmol Vis Sci. 2004;45(8):2606-12.

19. Huang XR, Bagga H, Greenfield DS, Knighton RW. Variation of peripapillary retinal nerve fiber layer birefringence in normal human subjects. Invest Ophthalmol Vis Sci. 2004;45(9):3073-80.

20. Zangwill LM, Bowd C, Berry CC, Williams J, Blumenthal EZ, SanchesGaleana CA, et al. Discriminating between normal and glaucomatous eyes using the Heidelberg Retina Tomograph, GDx Nerve Fiber Analyzer, and Optical Coherence Tomograph. Arch Ophthalmol. 2001;119(7):985-93. Comment in: Arch Ophthalmol. 2001;119(7):1069-70.

21. Medeiros FA, Zangwill LM, Bowd C, Weinreb RN. Comparison of the GDx VCC scanning laser polarimeter, HRT II confocal scanning laser ophthalmoscope, and stratus OCT optical coherence tomograph for the detection of glaucoma. Arch Ophthalmol. 2004;122(6):827-37.

22. Bourne RR, Medeiros FA, Bowd C, Jahanbakhsh K, Zangwill LM, Weinreb $\mathrm{RN}$. Comparability of retinal nerve fiber layer thickness measurements of optical coherence tomography instruments. Invest Ophthalmol Vis Sci. 2005;46(4): 1280-5. 